\title{
SOEP
}

SOEPpapers

SOEPDa Paers
on Multidisciplinary Panel Data Research

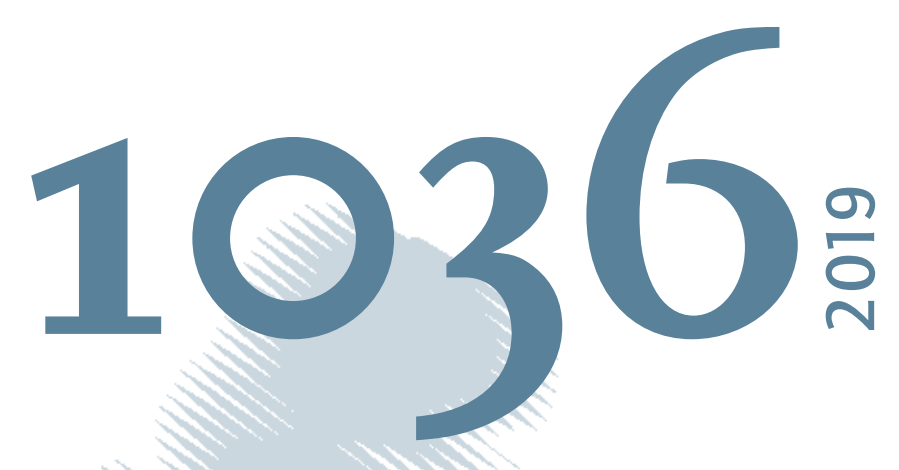

\section{Capturing Affective Well-Being in Daily Life with the Day Reconstrution Method: A Refined View on Positive and Negative Affect}


This series presents research findings based either directly on data from the German SocioEconomic Panel (SOEP) or using SOEP data as part of an internationally comparable data set (e.g. CNEF, ECHP, LIS, LWS, CHER/PACO). SOEP is a truly multidisciplinary household panel study covering a wide range of social and behavioral sciences: economics, sociology, psychology, survey methodology, econometrics and applied statistics, educational science, political science, public health, behavioral genetics, demography, geography, and sport science.

The decision to publish a submission in SOEPpapers is made by a board of editors chosen by the DIW Berlin to represent the wide range of disciplines covered by SOEP. There is no external referee process and papers are either accepted or rejected without revision. Papers appear in this series as works in progress and may also appear elsewhere. They often represent preliminary studies and are circulated to encourage discussion. Citation of such a paper should account for its provisional character. A revised version may be requested from the author directly.

Any opinions expressed in this series are those of the author(s) and not those of DIW Berlin. Research disseminated by DIW Berlin may include views on public policy issues, but the institute itself takes no institutional policy positions.

The SOEPpapers are available at http://www.diw.de/soeppapers

\section{Editors:}

Jan Goebel (Spatial Economics)

Stefan Liebig (Sociology)

David Richter (Psychology)

Carsten Schröder (Public Economics)

Jürgen Schupp (Sociology)

Sabine Zinn (Statistics)

Conchita D'Ambrosio (Public Economics, DIW Research Fellow)

Denis Gerstorf (Psychology, DIW Research Fellow)

Katharina Wrohlich (Gender Studies)

Martin Kroh (Political Science, Survey Methodology)

Jörg-Peter Schräpler (Survey Methodology, DIW Research Fellow)

Thomas Siedler (Empirical Economics, DIW Research Fellow)

C. Katharina Spieß (Education and Family Economics)

Gert G. Wagner (Social Sciences)

ISSN: 1864-6689 (online)

German Socio-Economic Panel (SOEP)

DIW Berlin

Mohrenstrasse 58

10117 Berlin, Germany

Contact: soeppapers@diw.de

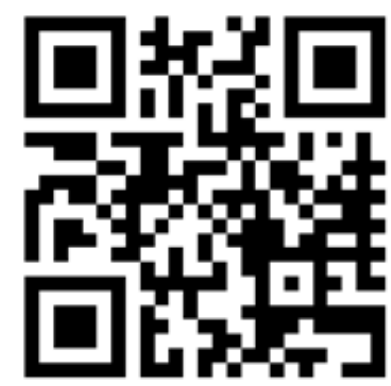




\title{
Capturing Affective Well-Being in Daily Life with the Day Reconstruction Method: A Refined View on Positive and Negative Affect
}

\author{
Dave Möwisch $^{1} \cdot$ Florian Schmiedek $^{1} \cdot$ David Richter $^{2} \cdot$ Annette Brose $^{3}$
}

\begin{abstract}
In the last years, there has been a shift from traditional measurements of affective well-being to approaches such as the day reconstruction method (DRM). While the traditional approaches often assess trait level differences in well-being, the DRM allows examining affective dynamics in everyday contexts. The latter may ultimately explain why some people feel more happy than others (e.g., because they experience more gratification during everyday experiences). Even though DRM research has increased in the last years, little is known about the structure of affective well-being in everyday life, and potential structural differences of affect at the within- and between-person level have rarely been considered. We thus thoroughly examined the structure of affective well-being in daily life, using data from a nationally representative sample $(N=2401)$ of the German Socioeco-nomic Panel Innovation Sample that were obtained with the DRM. Multilevel structural equation models revealed that (1) affective well-being in daily life cannot be reduced to the two global dimensions positive and negative affect (PA and NA) but that the structure of NA is more nuanced; (2) the emerging subfacets of NA have distinct associations with global indicators of well-being (e.g., life satisfaction); (3) there are structural differences of affective well-being at the within- and between-person level, and (4) the relationships between affect subfacets and activities such as "work" can be opposed at the within- and between-person level. These results show that a more differentiated view on the structure of affect contributes to a better understanding of affective well-being in everyday life.
\end{abstract}

Keywords Day reconstruction method - Positive affect $\cdot$ Negative affect $\cdot$ Multilevel structural equation modeling $\cdot$ Subjective well-being

This manuscript is published as:

Möwisch, D., Schmiedek, F., Richter, D., Brose A. (2019). Journal of Happiness Studies, 20(2), pp. 641-663. https://doi.org/10.1007/s10902-018-9965-3.

First published online: February 28, 2018

(C) Springer Science+Business Media B.V., part of Springer Nature 2018

Dave Möwisch

moewisch@dipf.de

1 German Institute for International Educational Research (DIPF), Warschauer Straße 34-38, 10243 Berlin, Germany

2 German Institute for Economic Research (DIW Berlin), Mohrenstraße 58, 10117 Berlin, Germany

3 Department of Psychology, Humboldt-Universität zu Berlin, Rudower Chaussee 18, 12489 Berlin, Germany 


\section{Introduction}

One essential way of quantifying individual differences in the quality of life is to ask people about their subjective well-being (SWB). Measures of SWB commonly ask people once for global evaluations of their lives (e.g., how satisfied one is with their life as a whole) or of affective well-being across expanded periods of time (e.g., how happy one was feeling throughout the last year) (Diener et al. 2002). An underlying assumption of such assessment procedures is that individuals' evaluations of well-being reflect trait-like individual differences that can be related to other aspects of quality of life such as health or educational background (Hudson et al. 2017).

Next to this more traditional approach to well-being, new strands of research, in the following referred to as the umbrella term experience sampling method (ESM), focus on more dynamic aspects of how people feel. Diary methods are used, for example, to examine how people manage the ups and downs of daily life including affective wellbeing (Bolger et al. 2003). Furthermore, the day reconstruction method (DRM; Kahneman et al. 2004) places particular emphasis on the assessment of daily activities and how they relate to momentary well-being.

The reasons for this shift are manifold-for example, the ecological validity of the data collected with ESM is compelling because the assessments are made close to realtime and in contexts people actually live in (Diener and Tay 2014). Furthermore, the methods seem promising in that they may point at mechanisms to why some people feel happier than others because the repeated measurements provide a window into withinperson dynamics (Shiffman et al. 2008).

The purpose of this study is to illuminate an important aspect in the context of ESM approaches to affective well-being that has very rarely been taken into consideration to date. Many studies treated affective well-being as a two-dimensional construct (positive and negative affect, PA and NA) and thus disregarded that phenomenologically more nuanced and specific affective states that may characterize everyday activities. We therefore examine how a more fine-grained view on affective well-being can enhance our understanding of subjective well-being and its correlates. We depart from a recent study that investigated affective well-being in everyday life via the DRM (Anusic et al. 2017). This study is exceptional in that the DRM was applied in a nationally representative sampling of German households and to an impressive number of participants. This study demonstrated clearly how PA and NA were related to daily activities, social partners, and global indicators of well-being. We took this work an important step further by suggesting an alternative representation of PA and NA. Aim 1 of our study is to present a model of affect that distinguishes between subfacets of NA and only integrates these subfacets at a higher level. Aim 2 is to distinguish between affective well-being at the trait level and the state level of within-person dynamics. Aim 3 is to demonstrate how this more fine-grained view on affective well-being can increase the predictive validity of affect both at the trait and state level, and thus helps understanding how daily activities (e.g., working) and global aspects of well-being (e.g., satisfaction with work) co-occur with specific affective qualities (e.g., stress, boredom). 


\subsection{Measuring Affective Well-Being in Everyday Life}

In the past, several methods were developed to approach affective well-being within persons over time. Diary studies (e.g., Zelenski and Larsen 2000) and the experience sampling method (ESM; Csikszentmihalyi and Larson 1987) are likely the most commonly used methods. Both are useful tools to investigate within-person changes over time as well as individual differences therein and the causes of change (Bolger et al. 2003). The ESM commonly includes self-reports of various constructs at time-based, event-based, or (quasi-) random sampling occasions in daily life (Bjälkebring et al. 2016; Stoeber and Janssen 2011). The ecological validity of this method is particularly high and retrospective biases are rather low (e.g., Hogarth et al. 2007; Engeser and Baumann 2016). Diary studies include retrospective self-reports which usually refer to longer periods than the ESM.

The specific feature of the DRM (Kahneman et al. 2004) is that it combines elements of ESM and diary studies. It comprises the structuring of the previous day into episodes and the assessment of affective well-being in these episodes. In contrast to the ESM, events are not sampled at random but nearly all activities of one reconstructed day are assessed which allows a more complete representation of peoples' lives. Additionally, the DRM may collect information about the location, other persons, and activities during the episodes, which provides important context information. For example, Oishi et al. (2011) showed that retired people feel happier when they are in a familiar environment rather than in an unfamiliar one. Furthermore, the DRM revealed diurnal rhythms for different aspects of mood (Stone et al. 2006). Positive feelings showed a bimodal pattern with peaks at noon and evening, whereas for negative emotions, peaks were found at mid-morning and midafternoon. Yet, another study revealed that aggregates of affective well-being obtained with the DRM correlate with more global measures of SWB (Diener et al. 2012), speaking for the some convergent validity of DRM data. In sum, the DRM has been shown to be a useful and valid tool to capture between-person differences and within-person fluctuations in affective well-being in everyday life.

Very recently, the validity and usefulness of the DRM was examined with data from a highly exceptional, large-scale study and with randomly sampled daily episodes: a nationally representative sample of German households (Anusic et al. 2017). The authors used data from 2401 participants of the German Socioeconomic Panel Innovation Sample (SEOP-IS, Richter and Schupp 2015) that were collected by means of a shortened version of the DRM developed by Kahneman et al. (2004). Participants evaluated their affective states on 13 items and for three randomly selected episodes. Three items were aggregated into a PA score and five items into an NA score by Anusic et al. (2017). The remaining items were examined separately. In addition, participants classified their activities during the episodes in accordance with categories such as "watching TV", "working", or "relax". Thus, Anusic et al. (2017) could analyze affective characteristics for each class of activities. For example, "doing sports" had the highest level of PA and "working/studying" the highest level of NA in the sample. Moreover, daily aggregates of the DRM were investigated in relation to global measures of well-being, such as life satisfaction. PA correlated with different indicators of global well-being. Surprisingly, there was no significant link between NA and life satisfaction; NA in daily life was only associated with a trait measure of NA. Overall, the study by Anusic et al. (2017) provides evidence on the validity of the DRM, in particular the random sampling approach, and it provides interesting findings on differential relationships between PA and NA in daily life as well as trait variables. 


\subsection{This Study}

The purpose of this study is to thoroughly examine the structure of affective well-being in daily life and in relation to activities and trait-level individual differences. We depart from the study of Anusic et al. (2017) that was just portrayed in detail, and we exemplarily conduct analyses that are, in principle, similar to theirs (albeit performed as structural equation models). Yet, we suggest and test an alternative, more differentiated representation of particularly NA. We propose that this representation will reveal new insights into affective well-being and might clarify unresolved issues that were obtained in previous studies on daily affect and its correlates (e.g., the non-significant association between NA as obtained from the DRM and life satisfaction; Anusic et al. 2017).

We could have based our research on other studies that have used the DRM. However, those have been conducted with smaller, non-representative samples and have dealt with more specific questions concerning daily well-being, such as work status (e.g., Knabe et al. 2010), personality (Oerlemans et al. 2011), or other context variables (Bakker et al. 2013; Nelson et al. 2013). Thus, departing from Anusic et al. (2017) and using the same data will likely provide the most generalizable findings.

To be more precise, the first aim of this study is to present a model of affect that distinguishes between subfacets of NA and integrates these subfacets at a superordinate level. Anusic et al. (2017) aggregated three items of PA (happiness, satisfaction, and enthusiasm) and five items of NA (anger, frustration, mourning, worries, and stress) and used these composite scores rather than single items, which is a commonly used approach in previous DRM research. The remaining items (fatigue, meaning, pain, boredom, and loneliness) were examined separately. This approach is widely used, also in order to calculate alternative affect indicators like net affect (Bradburn 1969) or the U-index (e.g., Kahneman et al. 2004; Knabe et al. 2010). However, the computation of composite scores that reduce various subfacets of affect to global dimensions has been criticized for obscuring important information about affective well-being (Schimmack 1999). For example, at a funeral, NA likely reflects mainly sadness whereas NA at work often reflects stress or frustration. That is, the same aggregated NA score may have different meanings depending on the situation, and the more diverse the affect items are, that are used in the computation of composite scores, the less it is known about the content of an aggregate score. In the case of the NA items available in our study, it is obvious that they are phenomenologically different. In view of dimensional models of affect (e.g., Russell 2003), they should differ in terms of activation (e.g., stress is high in activation whereas loneliness is rather low in activation). Furthermore, appraisal theorists have supported the idea of a more differentiated structure of affective states by defining affective states as a result of a sequence of appraisals (Lazarus 1991; Scherer 2001). Hence, the different items likely characterize different emotional episodes (Russell and Feldman Barrett 1999) and are the result of differentiable appraisal processes (e.g., mourning is a prototypical emotion in the context of loss experiences whereas frustration may indicate external control beliefs).

To address this problematic aspect of global composite scores, we examine a more differentiated structure of NA in accordance with the proposal of Watson and Tellegen (1985). In their theoretical framework, PA and NA can be interpreted as general dimensions. These are, however, superordinate to more circumscribed affective experiences. Accordingly, a model with lower-order factors below PA and NA allows distinguishing between situation-specific kinds of affect, such as sadness at a funeral and anger at 
work. In summary, we hypothesize that a model with multiple lower-level NA factors will have a better fit (i.e., represent the data better) than a model with only one NA factor that resembles the composite NA scores commonly computed in the literature. We did not have explicit hypotheses on the nature of the lower-order NA factors and used exploratory factor analysis to gain insights on those. While in principle similar hypotheses could be formulated for PA, we cannot test those with data from the SEOPIS because PA was assessed with only three items.

The second aim of this study is to distinguish between affective experiences and their structure at the trait level (between-person differences) and at the state level (within-person variation). In their study, Anusic et al. (2017) aggregated PA and NA items for each participant to duration-weighted indices. This often used procedure enables using mean levels of affective well-being over the day for each participant. However, the disadvantage of this procedure is that it potentially overlooks different structural relationships between facets of affect at the within- and between-person level (e.g., Hamaker 2012; Molenaar and Campbell 2009). In line with the literature, we propose here that the between-person correlations among different affect facets of the same valence are larger than the corresponding within-person correlations (Brose et al. 2015; Vansteelandt et al. 2005). The reason is that in a given situation, affective states of the same valence but from different facets often do not co-occur within persons. For example, situations eliciting anger (e.g., being insulted by someone) are often not the same that elicit worry (e.g., hearing about the illness of a close other). In contrast, at the level of between-person differences, different facets of affect when rated across different situations correlate more strongly because the ratings in part reflect stable tendencies to experience positive and negative feelings more generally. These propositions have found empirical support, for example in the context of an ESM study in which the structure of affect at the between-person level was in accordance with a dimensional model of affect whereas at the within-person level, a discrete model with more facets seemed to be more appropriate (Vansteelandt et al. 2005; cf. Brose et al 2015; Zelenski and Larsen 2000). That is, the various facets of affect such as anger, sadness, or anxiety are more distinguishable at the within-person level while at the between-person level, these affects tend to be correlated more strongly. As a consequence, affect items can be aggregated to higher-order dimensions at the between-person level (e.g., NA or PA). This should, however, not be the case for the within-person level where the specific content of affective well-being matters.

Overall, there is compelling evidence for the necessity to investigate the structure of affect separately at the between- and within-person level. Thus, we model affect within and between persons separately via multilevel modelling to identify possible structural differences between the two levels. We hypothesize that model parameters (i.e., factor loadings and factor correlations) of the within-person structure deviate from model parameters of the between-person structure.

The third aim of this study is to demonstrate how this more fine-grained view on affective well-being increases the predictive validity at the within- and between-person level. To this end, and as a replication of the results of Anusic et al. (2017), we examine the predictive validity of the NA subfacets with regard to (a) other indicators of well-being and (b) situational contexts. Regarding other indicators of well-being, we expect that the subfacets of NA have different associations at the between-person level with life satisfaction and indicators of trait NA/PA than a global factor. Given that we take an exploratory approach to the lower order NA factors, we can only formulate tentative assumptions on this issue. Yet, it seems that some high arousal NA states such as stress often occur in the context of challenge stressors whereas other states such as mourning occur in the context 
of hindrance stressors (for the distinction of these stressor types, cf. LePine et al. 2005). Challenge stressors may indicate a potential for learning as well as personal growth and often occur in the context of work, whereas hindrance stressors may indicate blocked goals or inevitable losses. It seems that those emotions that are indicative of the latter should be related to lower levels of well-being. Emotions that may indicate challenge or engagement may, on the other hand, be associated with higher levels of well-being.

Finally, we investigate the relationships of the NA subfacets with specific activities at the within- and between-person level. We assume that these relationships differ with regard to the level of investigation. For example, within-person associations between the NA subfacets and specific activities, such as "working", may be positive while they are negative at the between-person level. Such a finding would underline the utility and appropriateness of a separate modelling of affect in everyday life at the within- and between-person level.

\section{Method}

\subsection{Participants and Procedure}

The current study used data from the SOEP-IS (Richter and Schupp 2015; Anusic et al. 2017), which constitutes a nationally representative sample of the German population. The particular sample is a subsample of the larger SOEP and was drawn with the goal to introduce innovative methodological approaches, such as the DRM. As part of the data collection, German households were interviewed, including all household members who had reached at least the age of 16 . The sample includes a total of 2401 participants $(52 \%$ women) who were interviewed at least once in 2012 or 2013 (1721 participants were interviewed in 2012 and 2013; 513 only in 2012; and 167 only in 2013). Thus, the number of observations that participants contribute to this study varies (see below for details). The age of the participants ranged from 17 to 96 , with an average of 52.7 years ( $\mathrm{SD}=17.6$ years). The data collection was carried out by Computer Assisted Personal Interviews (CAPI) conducted by trained interviewers. The interview referred to the day previous to the day of the interview.

\subsection{Measures}

\subsubsection{DRM}

The DRM is an assessment of everyday activities and ratings of how persons feel during the activities (Kahneman et al. 2004). In this study, the participants were first asked when they had woken up. Subsequently, they were asked to describe what they had done next by using a list of 24 possible activities such as "work". This procedure was repeated until the entire day was reconstructed for 1 day per wave. Additionally, they stated starting and ending times of the episodes, interaction partners like friends, partners, or superiors during the episodes, and where they had been (at home, at work, or somewhere else). On average, 10.8 episodes per day were reported $(\mathrm{SD}=4.0)$. The duration of an episode averaged $88 \mathrm{~min}(\mathrm{SD}=118 \mathrm{~min}$ ). For further descriptive details see Anusic et al. (2017). In Fig. 1, an example of a reconstructed day of one participant is shown.

Essential for the present investigation, participants rated their experienced affect during the episodes. The affect ratings were conducted for three randomly selected episodes 


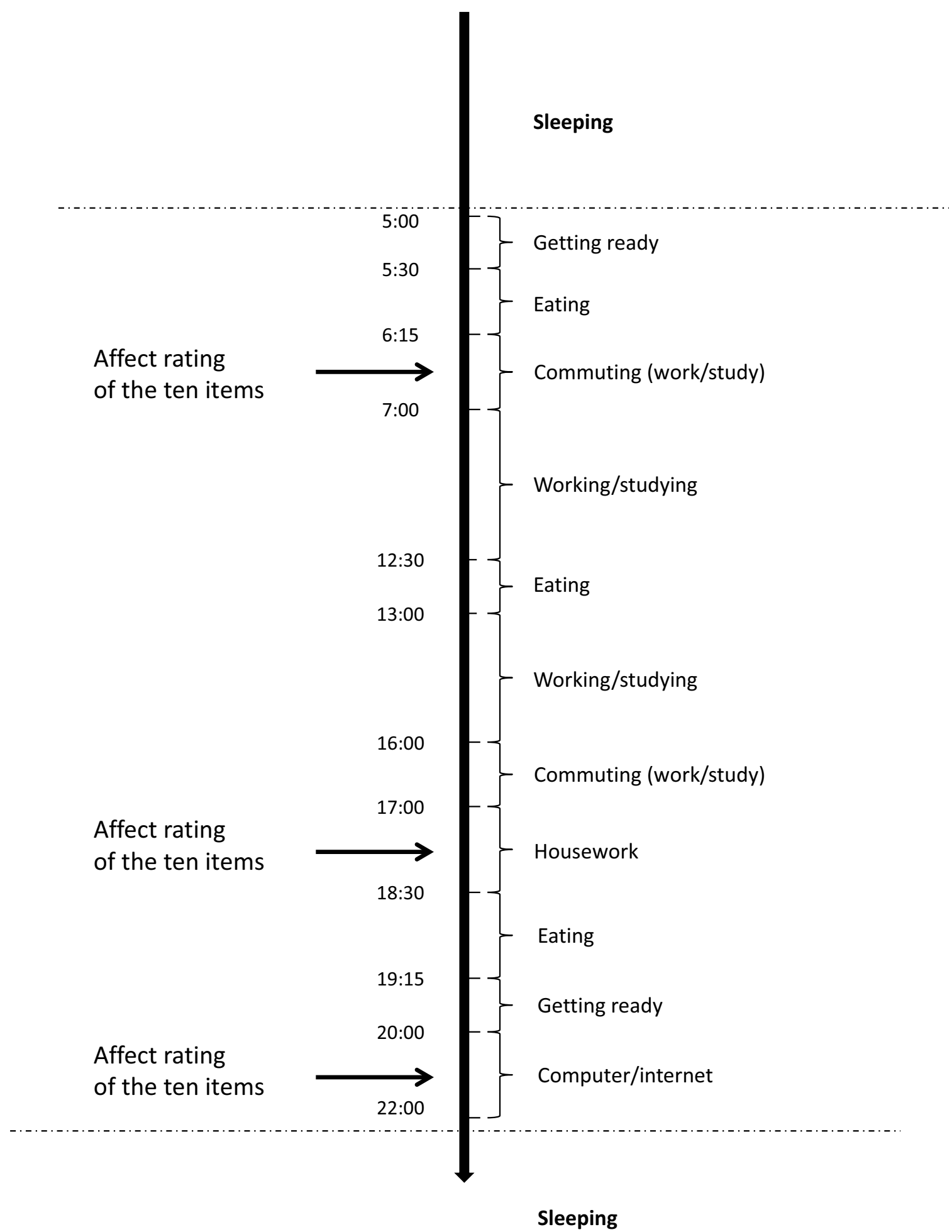

Fig. 1 Example of one reconstructed day via the DRM and three randomly selected episodes with an affect rating

of each participant. The three random episodes were selected by the CAPI program. Because some participants took either part in both or only in one of the waves, the number of assessed episodes regarding affect ranges from three to six episodes per participant. The following 13 items were rated for the three selected episodes (per person and occasion): happiness, enthusiasm, satisfaction, anger, frustration, stress, boredom, loneliness, 
mourning, worries, fatigue, pain, and deeper meaning. The scale ranged from 1 (not at all) to 7 (very much).

\subsubsection{Life Satisfaction}

General and domain-specific life satisfaction was measured for each study wave (2012 and 2013). The survey for life satisfaction and the trait variables of affect (see below) was part of the yearly interview of the SOEP-IS, which was conducted independently from this study and for each person and household. For general life satisfaction, a single item asked "How satisfied are you with your life, all things considered?". The domain-specific items were life satisfaction with one's own work and one's sleeping behavior. All single items were answered on an 11-point scale ranging from 0 (completely dissatisfied) to 10 (completely satisfied).

\subsubsection{Trait Affect}

Apart from the affective ratings of the daily episodes, the emotions experienced in the 4 weeks prior to the interview were surveyed. Trait PA was measured with the item happiness. Trait NA was assessed with four items (anger, worry, sadness, and gloomy). All five items were assessed with the instruction "Please specify for each how frequently or infrequently you have experienced this feeling in the last 4 weeks". For the items "happiness", "anger", "worry", and "sadness" the respondents used a 5-point Likert-type scale $(1=$ very rarely to $5=$ very often). For the item "gloomy" the respondents used a 5-point Likert-type $(1=$ always to $5=$ never $)$. This item was reversely coded for the analyses.

\subsection{Statistical Analyses}

First, we excluded the items fatigue, pain, and deeper meaning from the analysis. We excluded fatigue because Anusic et al. (2017) could show a W-shaped pattern of fatigue over the course of the day. Thus, the measurement of "fatigue" is confounded with the time of the day which we wanted to avoid. The item "pain" was excluded because pain as affective experience has commonly not been considered in empirical approaches to the structure of affective experiences (Russell 2003; Scherer 2001; Watson and Tellegen 1985). "Deeper meaning" was excluded because it corresponds to a cognitive evaluation rather than an affective state. The remaining ten affect items were analyzed in the MCFA.

Second, intraclass correlation coefficients (ICC) were computed for the 10 affect items. The ICC indicates the ratio of between-subject variance to overall variance of a variable. Small values reveal that overall variance is dominated by within-person fluctuations.

To test Aim 1 (to evaluate a model of affect that distinguishes between subfacets of NA) and Aim 2 (to distinguish between affective well-being at the trait and the state level of within-person dynamics), we conducted a set of multilevel confirmatory factor analyses (MCFA) using Mplus 7.4 (Muthén and Muthén 1998-2015). Multilevel analyses specify a model on different levels, taking into account the dependence of the observations introduced by clustered sampling or repeated assessments. In this study, the daily episodes (within level) are clustered in participants (between level). In particular, we applied multilevel structural equation models (multilevel SEM) which allow separating true variance at the between- and within-person level from (a) measurement error and item-specific variance at the within level (i.e., variance across different affect 
ratings of each episode) and from (b) sampling error at the between level that results from the aggregation of a limited number of only three episodes per day (i.e., doublylatent models; see Lüdtke et al. 2008; Marsh et al. 2009). Models were estimated using robust maximum-likelihood (MLR) which uses parameter estimates with standard errors and a Chi square statistic that are robust to non-normality and non-independence of observations. Missing data were managed with a full-information maximum likelihood approach (FIML). We evaluated the model fit using the $\chi^{2}$-values, comparative fit index (CFI), root mean square error of approximation (RMSEA), and standardized root mean square residual (SRMR). We applied the conventional cut-off criteria by $\mathrm{Hu}$ and Bentler (1999), that is, .95 or higher for CFI, .08 or lower for SRMR, and .06 or lower for RMSEA.

To approach Aim 3 (the examination of relationships between global NA versus specific NA facets on the one hand and life satisfaction and domain-specific satisfaction on the other hand), we conducted correlation and multiple regression analyses via multilevel SEM (details are reported together with the results). Finally, to pinpoint the usefulness of the separation of the within- and the between-person level, the subfacets of NA were regressed on dummy-coded variables for the activities "work" and "housework". The dummy-coded variables compared the specific activity (coded as 1) with all other activities (coded as 0 ). These results were compared with results from regression analyses that consider the dependence of the observations but do not specify separate models, and thereby do not allow for structural differences, at the within and betweenperson level (via Mplus; type = complex).

\section{Results}

\subsection{Affect Structure}

Table 1 shows the ICCs and the descriptive statistics of the 10 affect items. The ICCs of all affect items range from .22 to .41 indicating that differences within persons across situations dominated the overall variance of the affect ratings.

Table 1 Descriptive statistics of the ten affect items

\begin{tabular}{llccc}
\hline Variable & Mean (SD) & Skewness & Kurtosis & ICC \\
\hline Happiness & $3.93(1.86)$ & -.15 & -.08 & .34 \\
Enthusiasm & $3.08(1.85)$ & .39 & -.99 & .31 \\
Satisfaction & $4.27(1.74)$ & -.37 & -.62 & .32 \\
Anger & $1.16(1.13)$ & 2.22 & 4.63 & .22 \\
Frustration & $1.28(1.08)$ & 2.55 & 6.44 & .23 \\
Stress & $1.60(1.26)$ & 2.23 & 4.35 & .28 \\
Mourning & $1.27(.80)$ & 3.63 & 14.47 & .35 \\
Worries & $1.51(1.08)$ & 2.39 & 5.49 & .38 \\
Boredom & $1.51(1.09)$ & 2.43 & 5.78 & .35 \\
Loneliness & $1.40(1.02)$ & 3.02 & 9.37 & .41 \\
\hline
\end{tabular}

$S D$ standard deviation, $I C C$ intraclass correlation 


\subsection{Aim 1: A Differentiated Model of Negative Affect}

To approach Aim 1 (to evaluate a model of affect that distinguishes between subfacets of NA) and Aim 2 (to distinguish between affective well-being at the trait and the state level of within-person dynamics) we carried out a set of MCFAs with different configurations of latent factors and model parameters. Table 2 presents model fit indices of these MCFAs. Regarding Aim 1, we postulated a model of affect that distinguishes between subfacets of affect and a second-order NA factor. In order to evaluate the appropriateness of such a model, a series of models (M1-M3) were compared. Please note that the factor loadings were estimated separately for the within- and the between-person level in Models 1-3.

Model 1 does not distinguish between subfacets of NA. The structure includes one latent factor for PA (three items) and one latent factor for NA (seven items). The items happiness, enthusiasm, and satisfaction load on the PA factor. Please note that this factor is the same across all subsequent models. The items anger, frustration, stress, mourning, worries, boredom, and loneliness load on the NA factor. In Model 2 (Fig. 2), we tested a more differentiated structure of NA consisting of three latent factors. The splitting into a threefactor structure for NA was based on preliminary multilevel exploratory factor analyses, which indicated that three NA factors and one PA factor at the within- and between-level represent the optimal number of latent factors for the ten items. The three NA factors were allowed to correlate in Model 2. Model 3 (Fig. 3) has the same first-order factors as Model 2 but also includes an additional second-order factor on which the three subordinate latent factors load.

The results of the model comparisons are the following. Of the three models, Model 2 showed the best model fit, followed by Model 3 and Model 1. To test whether differences in model fit were significant, we calculated the Satorra-Bentler scaled $\chi^{2}$-difference test (Satorra and Bentler 2001). The $\chi^{2}$ test statistics of all three models differed significantly (M2-M3: $\Delta \chi^{2}(4)=29.62, p<.05$; M3-M1: $\Delta \chi^{2}(6)=1151.37, p<.05$; M2-M1: $\Delta \chi^{2}$ $(10)=907.29, p<.05$ ). These results show that models with multiple NA factors (Models 2 and 3) provide a better representation for NA than the one-factor solution of NA (Model 1). Although Model 2 has a significantly better $\chi^{2}$ statistic compared to Model 3 , we still use both models in the subsequent analyses for two reasons. First, the other model fit indices (CFI, RMSEA, SRMR) showed acceptable values for both models. Second, using both models allows the comparison of the predictive validity of a model with several specific NA subfacets and a model with a global NA factor with regard to other indicators of wellbeing (Aim 3, see below).

\subsection{Aim 2: Differentiating Affect at the Within- and Between-Person Level}

Aim 2 of this study was to distinguish the structure of affective well-being at the trait level (between-person differences) and at the state level (within-person variation). Models 1-3 were instructive regarding this aim. In addition, similar models were tested, with the important difference that corresponding parameters were fixed across levels (Models 4-6). Only the models with a differentiated affect structure (Models 5 and 6) had an acceptable model fit. The fit was best for Model 5 which contained three subfacets of NA and no additional second order factor (Table 2). The $\chi^{2}$ test statistics of the three models (M4-M6) also differed significantly (M5-M6: $\Delta \chi^{2}(6)=55.19, p<.05$; M4-M6: $\Delta \chi^{2}(6)=1396.95$, $p<.05$; M5-M4: $\left.\Delta \chi^{2}(12)=1119.43, p<.05\right)$. To identify possible structural differences in 


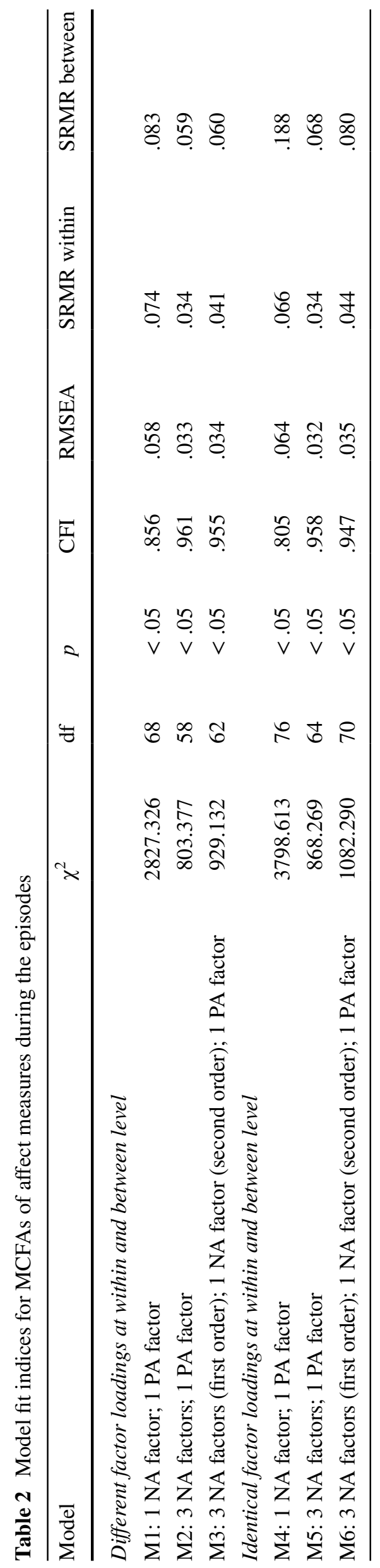




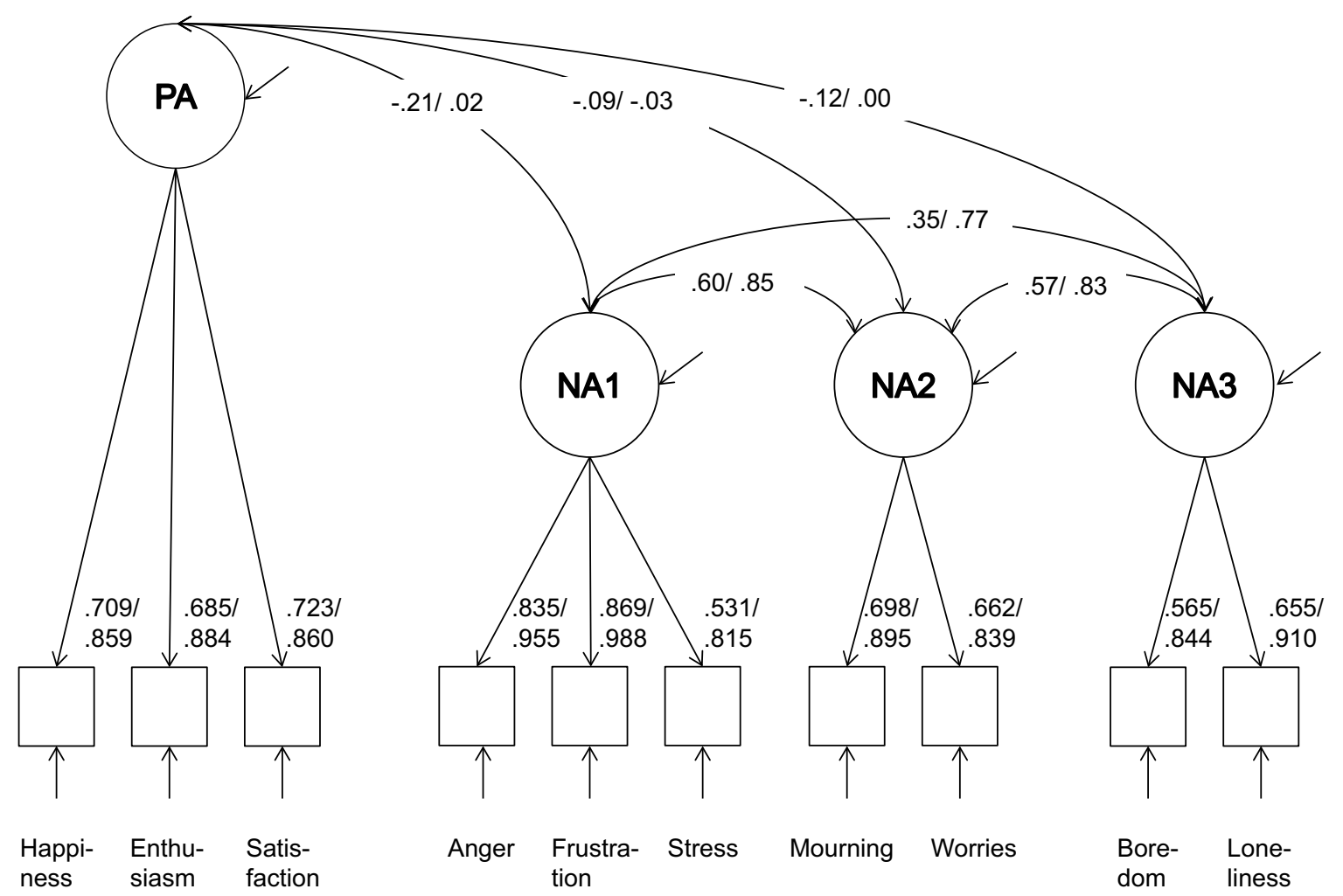

Fig. 2 Affect structure with three NA subfacets with standardized factor loadings and factor correlations (within-person level/between-person level)

variations between and within individuals, we compared two sets of models: M2 and M5 as well as M3 and M6 (i.e., the models with NA subfacets and with either non-fixed or fixed loadings across levels in the case of Models 2 and 3 and Models 5 and 6, respectively). In particular, it was tested whether the restriction of identical parameter values resulted in a significant increase in the $\chi^{2}$ test statistics. A significant increase in the $\chi^{2}$ test statistics for the models with identical parameter values across both levels (M5 and M6) would indicate structural differences at both levels. The constrained model (M5) fitted significantly worse $\left[\Delta \chi^{2}(6)=68.46, p<.05\right]$ than the model without constrained factor loadings across both levels (M2). In the comparison of M3 and M6, which both contained an additional second-order factor, the constrained model (M6) fitted significantly worse $\left[\Delta \chi^{2}(8)=145.83\right.$, $p<.05]$ than M3 with different factor loadings. Moreover, we tested the invariance of the latent correlation between PA and NA (second order) across both levels. The model with different latent correlations fitted significantly better $\left[\Delta \chi^{2}(1)=14.22, p<.05\right]$ than a model with equal latent correlations across the levels. In sum, the model with three subfacets and the model with three subfacets and a second-order factor fitted significantly better when factor loadings and latent correlations were different across the levels. These findings underline the structural distinction of affective well-being at the trait level (between-person differences) and at the state level (within-person variation).

\subsection{Aim 3: The Predictive Validity of a More Differentiated Affect Structure}

Aim 3 of this study was to demonstrate how a more differentiated view of affective wellbeing can increase the predictive validity at the within- and between-person level. In 


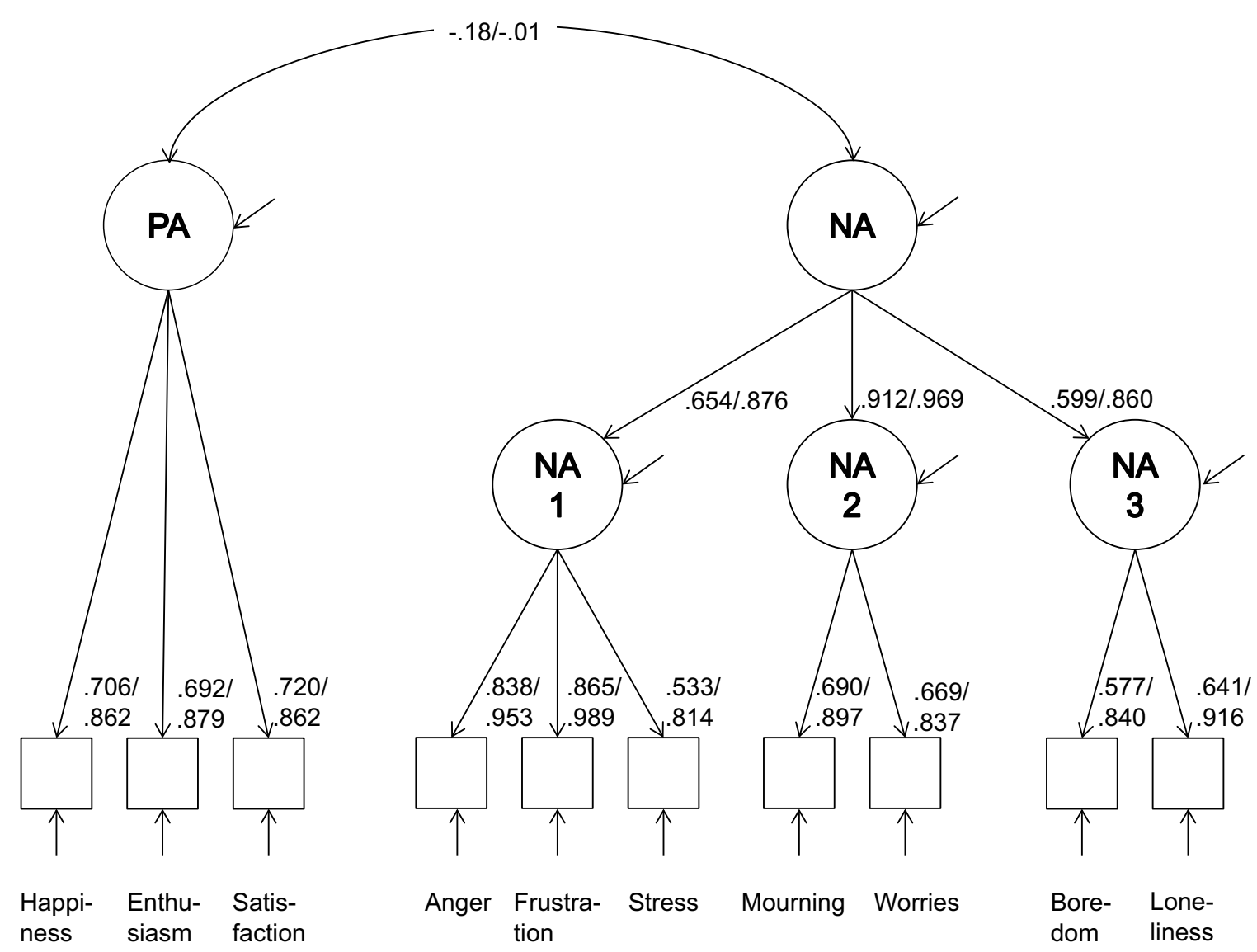

Fig. 3 Affect structure with three subfacets and a second-order factor for NA with standardized factor loadings and factor correlations (within-person level/between-person level)

particular, we tested how global versus differentiated NA factors predicted other indicators of well-being and how affect, that is modelled separately at the within- and between-person level, is distinctively related to daily activities working and housework in particular.

\subsubsection{Prediction of Other Indicators of Well-Being}

In our first series of analyses, we examined the predictive validity of the derived NA factors (i.e., the global NA factor and the three specific NA factors) for other indicators of well-being, such as life satisfaction, at the between-person level. For this purpose, we conducted correlation and multiple regression analyses. The latter were carried out as multilevel SEMs so we could work with the differentiated models of affect that were found to be superior in the context of Aims 1 and 2.

The pattern of simple correlations is presented in Table 3. The results show significantly positive correlations between PA and all indicators of well-being (except for Trait NA). All NA subfacets correlated significantly negatively with all indicators of well-being (except Trait PA).

When predicting the indicators of well-being with multiple regression analyses, we used two different models of NA. On the one hand, we used the model with only three NA subfacets and no higher-order factor, which had the best fit of all models (equal to Model 2 of the MCFAs; Fig. 2). On the other hand, we used the model with three subfacets and the second-order NA factor (equal to Model 3 of the MCFAs; Fig. 3). This enabled us to 


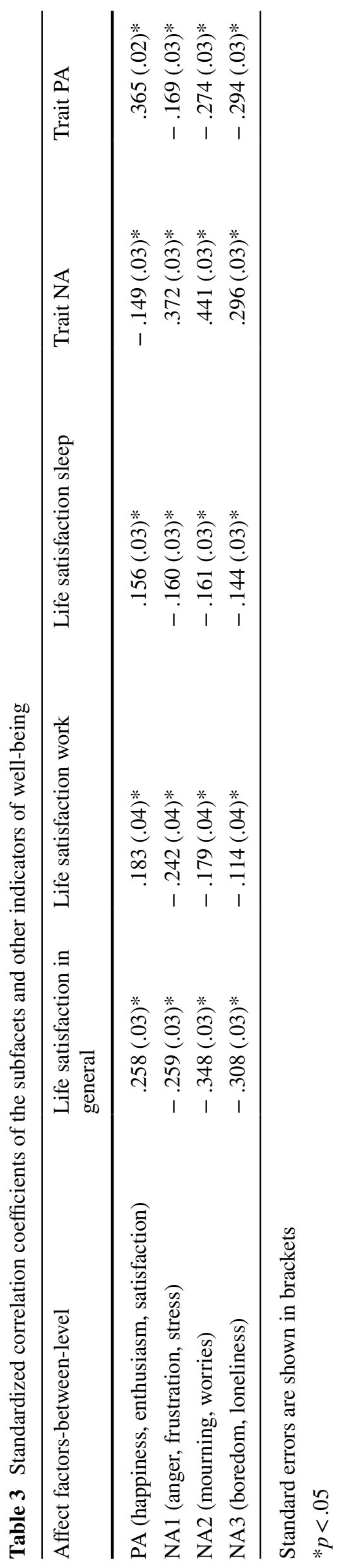


compare the predictive validity of the specific subfacets of NA and the global NA factor regarding other indicators of well-being. Put differently, the analyses with two different models of affect were done with the intention to use specific predictors (subfacets) in comparison to a more global measure of NA. In either case, the latent NA factors at the between-person level were modelled as predictors of well-being. The results of these analyses are presented in Table 4.

In the model with three NA facets, the participants' life satisfaction in general was positively associated with the PA factor, and NA2 (mourning, worries) was negatively associated with life satisfaction. Persons who experienced more happiness/enthusiasm/satisfaction and less mourning/worries in everyday life had a higher level of life satisfaction. The other two NA facets NA1 (anger, frustration, stress) and NA3 (boredom, loneliness) were not associated with life satisfaction over and above NA2. For satisfaction with respect to the participants' own work, there were significant effects for PA, NA1, and NA3. That is, persons with a higher level of happiness/enthusiasm/satisfaction and a lower level of anger/ frustration/stress in everyday life reported a higher level of work satisfaction. Contrary to expectations, more loneliness/boredom in everyday life were positively related to a higher work satisfaction. Satisfaction of one's own sleep was only significantly associated with PA. Thus, individuals with more happiness/enthusiasm/satisfaction during the day were individuals with a higher level of sleep satisfaction. Trait NA as outcome was negatively associated with PA and positively associated with NA2. That is, persons with less happiness/enthusiasm/satisfaction and more mourning/worries in everyday life reported more negative affect for the previous 4 weeks. Contrary to expectations, NA3 was negatively associated with Trait NA. However, the likelihood ratio test for this parameter was not significant, which means that the parameter was not reliably different from zero. Finally, Trait PA was positively associated with PA and, counter-intuitively, also with NA1. Persons who reported more anger/frustration/stress in the DRM also reported a higher frequency of happiness in the last 4 weeks. NA2 and also NA3 were negatively associated with Trait PA.

In the model with the higher-order NA factor (M3), the NA factor was significantly negatively associated with all life satisfaction variables and showed a positive relationship to Trait NA. Taken together, the way NA is conceptualized (either with or without a secondorder factor that is used for predictive purposes) provides distinct patterns of predictive validity on other indicators of well-being.

\subsubsection{Affective Characteristics of Specific Activities}

In the second series of analyses for Aim 3, we examined the associations between the subfacets of NA and the activities "working" and "housework" at the within- and betweenperson level. The purpose of these analyses was to underline the utility of the separate modelling of affect at these two levels of analysis. The activities "working" and "housework" were chosen exemplarily as in Anusic et al. (2017). These activities were mentioned most frequently in the interviews and they had the highest negative affect score (working) and lowest positive affect score (housework). The respective activity was operationalized via a dummy-coded variable that compares the specific activity (coded as 1) and all other activities (coded as 0). The regression analyses were again carried out as multilevel SEM, and the results of the two-level analyses are shown on the left-hand side of Tables 5 and 6. In addition to these analyses, we carried out regression analyses that took into account the dependence of the observations but neglected a separate modelling of affect at the between- and within-person level. These results are presented on the right-hand side of 


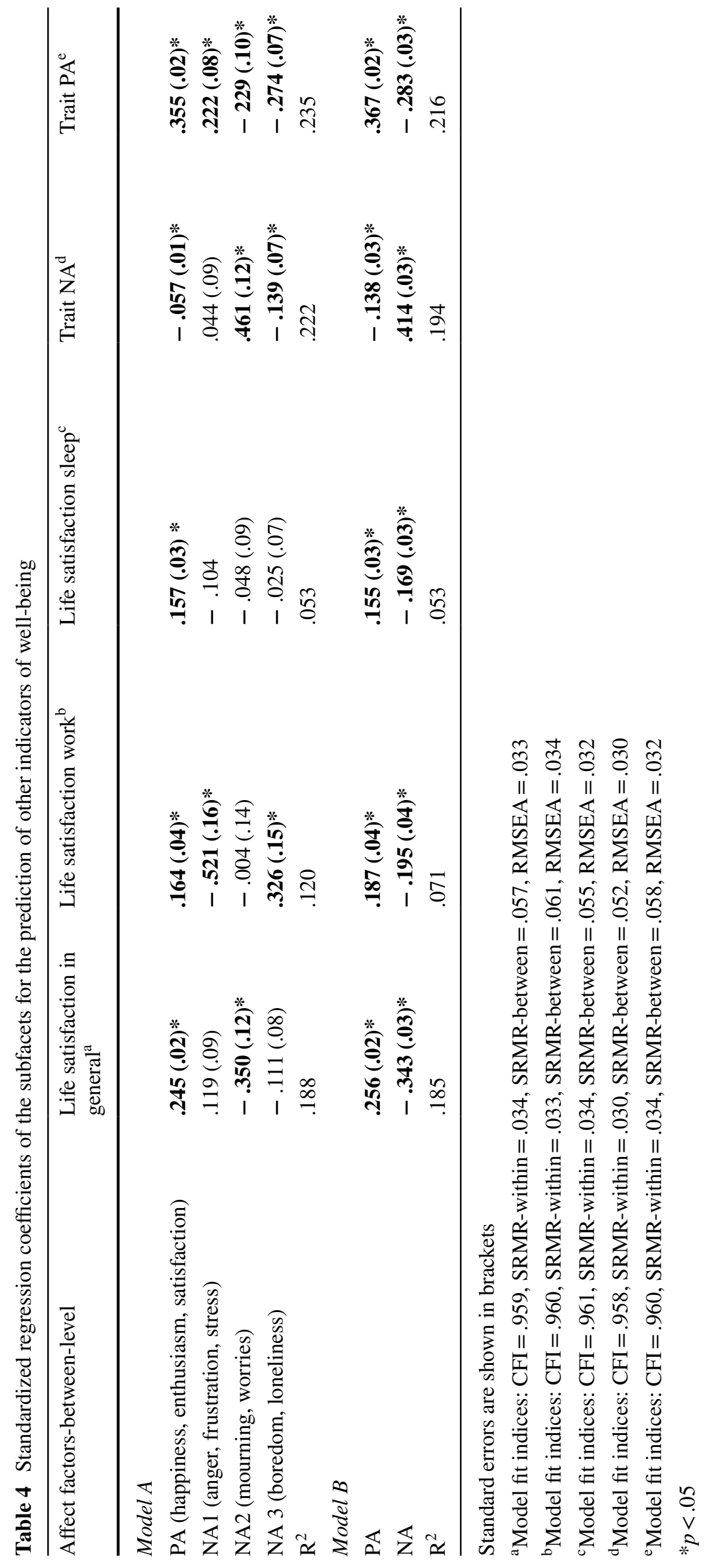


Table 5 Standardized regression coefficients of the activity "working" on the NA subfacets

\begin{tabular}{lrrr}
\hline & $\gamma$ & SE & $p$ \\
\hline Within & & & \\
PA (happiness, enthusiasm, satisfaction) & .000 & .01 & .97 \\
NA1 (anger, frustration, stress) & .245 & .02 & .00 \\
NA2 (mourning, worries) & .060 & .01 & .00 \\
NA3 (boredom, loneliness) & .009 & .02 & .56 \\
Between & & & \\
PA (happiness, enthusiasm, satisfaction) & -.006 & .06 & .93 \\
NA1 (anger, frustration, stress) & .158 & .08 & .03 \\
NA2 (mourning, worries) & -.082 & .06 & .18 \\
NA3 (boredom, loneliness) & -.079 & .06 & .19 \\
Type = complex & & & \\
PA (happiness, enthusiasm, satisfaction) & -.001 & .01 & .98 \\
NA1 (anger, frustration, stress) & .220 & .14 & .00 \\
NA2 (mourning, worries) & .031 & .20 & .01 \\
NA3 (boredom, loneliness) & -.006 & .11 & .57 \\
\hline
\end{tabular}

Model fit indices (type $=$ two-level): CFI $=.938$, SRMR-within $=.041$, SRMR-between $=.058, \mathrm{RMSEA}=.039$; model fit indices $($ type $=$ complex): $\mathrm{CFI}=.943, \mathrm{SRMR}=.041, \mathrm{RMSEA}=.039$

\begin{tabular}{lrrr}
\hline & $\gamma$ & $\mathrm{SE}$ & $p$ \\
\hline Within & & & \\
PA (happiness, enthusiasm, satisfaction) & -.167 & .01 & .00 \\
NA1 (anger, frustration, stress) & .048 & .01 & .00 \\
NA2 (mourning, worries) & -.032 & .01 & .01 \\
NA3 (boredom, loneliness) & .045 & .01 & .01 \\
Between & & & \\
PA (happiness, enthusiasm, satisfaction) & -.071 & .07 & .31 \\
NA1 (anger, frustration, stress) & -.196 & .08 & .01 \\
NA2 (mourning, worries) & .023 & .08 & .77 \\
NA3 (boredom, loneliness) & -.108 & .08 & .17 \\
Type = complex & & & \\
PA (happiness, enthusiasm, satisfaction) & -.134 & .01 & .00 \\
NA1 (anger, frustration, stress) & .022 & .01 & .03 \\
NA2 (mourning, worries) & -.020 & .01 & .04 \\
NA3 (boredom, loneliness) & .016 & .01 & .18 \\
\hline
\end{tabular}

Model fit indices $($ type $=$ two-level): $\mathrm{CFI}=.961$, SRMR-within $=.032$, SRMR-between $=.055, \mathrm{RMSEA}=.031$; model fit indices $($ type $=$ complex): $\mathrm{CFI}=.960, \mathrm{SRMR}=.032, \mathrm{RMSEA}=.042$ 
Tables 5 and 6. The two different approaches were used to illustrate the importance of our proposed within-between separation.

The activity "working" (Table 5, left side) was significantly positively associated with NA1 and NA2 at the within-person level. This means that people experienced more anger/frustration and mourning/worries during "working" than in other activities. On the between-person level, only NA1 was positively associated with "working". Thus, persons in "working" activities experienced more anger/frustration/stress than persons in other activities. When examining the relations without separating the levels of analysis (Table 5, right side), we found significant positive associations between NA1 and NA2 with the activity "working". Hence, the analysis that does not separate between the within- and the between-person level resembles the result from the within- but not the between-person level from the former model.

The activity "housework" (Table 6, left side) was significantly associated with all latent NA affect factors at the within-person level. While there were negative effects of "housework" on PA and NA2, the associations between "housework" on NA1 and NA3 were positive. Thus, persons experienced more anger/frustration/stress and boredom/loneliness during "housework" than in other situations. On the other hand, persons experienced less happiness/enthusiasm/satisfaction and mourning/worries during housework than in other activities. The analysis on the between-person level showed only a significant negative association for NA1. Persons who reported doing "housework" reported less anger/ frustration/stress than persons who reported other activities. This result showed a different directional association than in the analysis at the within-person level. Thus, for the activity "housework", a different association between the activity and one of the NA subfacets emerged at the within- and between-person level.

For the regression analysis without the within-between separation (Table 6, right side), there was a significantly negative association between "housework" and PA and NA2, and a significantly positive relationship between "housework" and NA1. In contrast to the multilevel regression with a separation of within- and between-person levels, persons who reported doing "housework" experienced more anger/frustration/stress than persons in other activities.

The results show that a different modelling of affect in everyday life between and within persons can yield different relations. Modelling affect without this separation can undermine differentially directed relationships at the within- and between-person level.

\section{Discussion}

The purpose of this study was to provide a more fine-grained view on affective well-being in everyday life than has been common in the literature. First, we proposed a model of affect that differentiates between subfacets of NA and thereby goes beyond prior research on affective well-being in daily life in which it has been common to compute global composite scores of PA and NA. Second, we investigated structural differences in daily affective well-being between the within-person and between-person level because the previous literature indicated different structural properties at both levels (Brose et al. 2015; Vansteelandt et al. 2005; Zelenski and Larsen 2000). Third, we examined the usefulness of the differentiation of (a) subfacets of NA and (b) the between- and the within-person level regarding relations to other variables. The results are in line with our expectations. A model that differentiates between various facets of NA was superior to a model with only one NA 
factor, with the latter being similar to a global composite score that is commonly computed in the literature. Moreover, we could show that there are structural differences between the within- and the between-person level, namely higher factor loadings at the betweenperson level and significantly different latent correlations between PA and the second-order NA factor. The additional analyses clearly pointed out the usefulness of our approach. The associations between the NA subfacets and life satisfaction were different from the association between global NA and life satisfaction. Furthermore, the relationships of the NA subfacets with specific activities (e.g., "housework") partly took different directions at the within- and between-person level. The results underline the relevance of a differentiated modelling of affect between individuals and within individuals across situations.

\subsection{Well-Being in Everyday Life}

One important implication of this study is the differentiated view of NA by our proposed factor structure of affective well-being in everyday life. Up to now, DRM research has, to our knowledge, only distinguished between PA and NA on a global level without subfacets. We postulated a more fine-grained structure of NA and found support for a second-order NA factor with three lower-level factors. In line with Watson and Tellegen (1985), the subfacets reflect specific characteristics of affective experiences, whereas the global factor represents the shared negative valence of all three subfacets. Moreover, and from the perspective of the model of core affect (Russell 2003) or the circumplex model of affect (Russell 1980), the first subfacet (NA1), measured by anger, frustration, and stress, is characterized by a high arousal. The second subfacet (NA2), based on the items mourning and worries, has a moderate level of arousal and reflects a high level of negative valence. Finally, and in accordance with appraisal theories (e.g., Lazarus 1991; Scherer 2001), cognitive processes such as evaluations and appraisals likely determined the specific affective states people reported, and these processes should underlie the differentiated NA factor structure. In particular, one possible mechanism for the separation of NA2 (loneliness and boredom) and NA3 (mourning and worries) is a lack of stimulation that is specific to the NA2 but not the NA3 factor. Boredom reflects a lack of internal and external stimulation in general (Struk et al. 2016) while loneliness may depict especially a lack of social stimulation. Mourning and worries on the other hand are not characterized by a lack of stimulation but often occur together with the experience of loss (mourning) or anticipated loss (worries). Together, the differentiated NA structure is in accordance with multiple theoretical accounts of affective experiences.

The relevance of this differentiated factor structure was shown in the analyses with other indicators of well-being. The correlation coefficients showed that both the specific subfacets and the global NA factor were useful correlates of life satisfaction and other trait variables. However, in the regression analyses, several complex results emerged. While the global NA factor was negatively related to all domains of life satisfaction, the subfacets showed a differentiated pattern. For general life satisfaction, NA3 was the only significant predictor in a model with all three subfacets. This important information could not have been obtained if NA had been conceptualized as a global composite score.

In a similar vein, an interesting finding emerged for work satisfaction. People who reported more anger, frustration, and stress (NA1) in everyday life reported less work satisfaction-a finding that was intuitively plausible. Interestingly, however, boredom and loneliness (NA3) in everyday life was positively related to work satisfaction in the same model. This surprising positive association might be due to a suppressor effect (Cohen et al. 2003) 
as the simple correlations showed positive associations between work satisfaction and all three subfacets of NA.

The results for sleep satisfaction indicate that the predictive variance of the three subfacets overlap; but only the second-order factor was significant, the subfacets, however, were not. Thus, general negative affectivity during the day seems to be crucial for sleep satisfaction and less so the specific subfacets of NA. Finally, the prediction of trait NA and PA also revealed a differentiated pattern of relationships for the NA subfacets and one contra-intuitive finding. NA1 was positively related to Trait PA. Persons who experienced more anger/frustration/stress in everyday life also reported a higher frequency of happiness in the previous 4 weeks. As the zero-order correlation between NA1 and Trait PA was negative, a possible suppressor effect within the subfacets might again provide an explanation for these findings.

In summary, a differentiated view of affect using the subfacets reveals insights that could not be obtained with a single NA factor. Importantly, however, it depends on the outcome variable whether a global measure or the subfacets show a better predictive validity.

An additional important aspect of these analyses is the following: The work by Anusic et al. (2017) our study follows up on did not show any significant associations between their composite score of NA and life satisfaction. This finding differs from our results according to which the second-order, global NA factor as well as the specific NA factors predict life satisfaction. This difference could be due to the fact that Anusic et al. (2017) did not include the item boredom when they computed their NA aggregate. It is also possible, however, that the difference across studies emerged because our study conceptualized global NA as a second order factor that reflects the specific aspects of NA to a varying degree (as indicated by the loading pattern). Thus, modelling NA at the latent level reveals associations that cannot be obtained from the unweighted computation of composite scores. Finally, an issue for further research remains whether to model specific or global indices of affective well-being in everyday life. Our results indicate that there is no simple answer to this question, as the predictive validity is neither consistently better for one or the other. It seems to depend on the outcome variable whether global or specific indices, reflecting either the common valence among the items or the specific content of emotional experiences, are more appropriate. This in itself is a noteworthy finding.

\subsection{Within- and Between Dynamics}

The second important implication of this paper concerns the separate modelling of affect at the within- and between-person level. Our results were consistent with previous research in demonstrating that there are structural differences across levels (Brose et al. 2015; Vansteelandt et al. 2005; Zelenski and Larsen 2000). In particular, the results provided evidence for different factor loadings across the levels (higher factor loadings at the between-person level) and a different latent correlation between NA and PA. While the latent correlation between PA and NA was negative at the within-person level, it did not differ significantly from zero at the between-person level.

The importance of the separate modelling of affect on both levels is underlined by the results of the regression analyses of the latent factors on the activities "work" and "housework". In particular, the direction of the association between "housework" and the subfacet NA1 was different on both levels. At the between-person level, people reported less anger, frustration, and stress (NA1) during housework activities than people who rated other activities. At the within-person level, however, people felt angrier and more frustrated 
and stressed (NA1) during housework than in other situations. This example illustrates that different associations can arise across the levels although the same variables are used. Thus, multilevel modelling should be considered for further investigations regarding affective well-being in everyday life. The corresponding analysis, which took into account the dependence of the observations but did not consider separate models of affect as the twolevel model, showed a positive relationship between "housework" and NA1, similar to our finding at the within-person level. The latter approach, which is similar to the usual approach in the previous research using DRM data, was not able to identify different associations between "housework" and NA1 across the levels. These results furthermore demonstrate the necessity to separate the investigation of relationships into a between and a within part.

\subsection{Limitations and Further Research}

There are some methodological and substantive limitations that should be considered. First, the data collection of the affect ratings only was conducted for three randomly selected episodes per person per year. Anusic et al. (2017) demonstrated the validity of the random-sampling DRM. Nevertheless, the reliability of the measurements must be viewed rather critically, in particular at the within-person level. Second, the operationalization of NA might not be sufficient. There are a variety of other affective states that have not been examined in this study. For example, anxiety, which plays a central role in many theories about affect, was not considered. The measurement of PA could also be reconsidered. A wider range of PA items should be included for further examinations since it is not possible to model subfacets on the basis of three PA items. Third, we have analyzed the aggregated within-person structure for all participants over 2 years and treated this as being informative about between-person differences. Individual structures, however, may differ from the aggregated within-person structure. Fourth, the reliabilities of the latent affect factors are lower at the within-person level $(\mathrm{PA}=.75, \mathrm{NA} 1=.79, \mathrm{NA} 2=.62, \mathrm{NA} 3=.54)$ than at the between-person level $(\mathrm{PA}=.90, \mathrm{NA} 1=.93, \mathrm{NA} 2=.85, \mathrm{NA} 3=.87)$, particularly so for NA3. This needs to be taken into account when interpreting within-person relations of these factors to other time-varying variables. Similar to the lower correlations among the affect factors, the lower correlations among the items of each affect factor may indicate a more differentiated affect structure at the within-person level. This may be further investigated using more items to represent each of the affect factors identified here, which may potentially allow a differentiation into further sub-factors (e.g., separable factors of boredom and loneliness). Fifth, the range of age is very large. This age heterogeneity leads to differences in time use and activities in everyday life (e.g., employment vs retirement) and affect. Studies showed that older adults with increasing age tend to experience less happiness (Bjalkebring et al. 2015) and less anger (Kunzmann et al. 2013). This confounding between age and affect should be taken into account in further investigations.

Further research should make use of the gained knowledge about a more differentiated structure of affective well-being in everyday life with subfacets of NA. These subfacets could be considered for further examinations with other relevant outcomes, such as educational background, personality traits, or situation-specific context variables, in order to extend our understanding of the causes and effects of affect in everyday life. Finally, multilevel modelling of affective well-being should also be given more attention, as there are indications for structural differences for within-person and between-person variations which may be essential for further research. 
Acknowledgements This research was funded by Grant NimoErt1 from the Federal Ministry of Education and Research (BMBF). We thank Eunji Lee for editorial assistance.

\section{References}

Anusic, I., Lucas, R. E., \& Donnellan, M. B. (2017). The validity of the day reconstruction method in the German socio-economic panel study. Social Indicators Research, 130(1), 213-232. https://doi.org/10.1007/ s11205-015-1172-6.

Bakker, A. B., Demerouti, E., Oerlemans, W., \& Sonnentag, S. (2013). Workaholism and daily recovery: A day reconstruction study of leisure activities. Journal of Organizational Behavior, 34(1), 87-107. https://doi. org/10.1002/job.1796.

Bjalkebring, P., Västfjäll, D., \& Johansson, B. E. A. (2015). Happiness and arousal: Framing happines as arousing results in lower happiness ratings for older adults. Frontiers in Psychology, 6, 706. https://doi. org/10.3389/fpsyg.2015.00706.

Bjälkebring, P., Västfjäll, D., Svenson, O., \& Slovic, P. (2016). Regulation of experienced and anticipated regret in daily decision making. Emotion, 16(3), 381-386. https://doi.org/10.1037/a0039861.

Bolger, N., Davis, A., \& Rafaeli, E. (2003). Diary methods: Capturing life as it is lived. Annual Review of Psychology, 54(1), 579-616. https://doi.org/10.1146/annurev.psych.54.101601.145030.

Bradburn, N. M. (1969). The structure of psychological well-being. Chicago: Aldine.

Brose, A., Voelkle, M. C., Lövdén, M., Lindenberger, U., \& Schmiedek, F. (2015). Differences in the betweenperson and within-person structures of affect are a matter of degree. European Journal of Personality, 29(1), 55-71. https://doi.org/10.1002/per.1961.

Cohen, J., Cohen, P., West, S. G., \& Aiken, L. S. (2003). Applied multiple regression/correlation analysis for the behavioral sciences. Mahwah, NJ: Lawrence Erlbaum.

Csikszentmihalyi, M., \& Larson, R. (1987). Validity and reliability of the experience-sampling method. Journal of Nervous and Mental Disease, 175(9), 526-536. https://doi.org/10.1097/00005053-198709000-00004.

Diener, E., Fujita, F., Tay, L., \& Biswas-Diener, R. (2012). Purpose, mood, and pleasure in predicting satisfaction judgments. Social Indicators Research, 105(3), 333-341. https://doi.org/10.1007/s11205-011-9787-8.

Diener, E., Lucas, R. E., \& Oishi, S. (2002). Subjective well-being: The science of happiness and life satisfaction. In C. R. Snyder \& S. J. Lopez (Eds.), The handbook of positive psychology (pp. 63-73). Oxford: Oxford University Press.

Diener, E., \& Tay, L. (2014). Review of the day reconstruction method (DRM). Social Indicators Research, 116(1), 255-267. https://doi.org/10.1007/s11205-013-0279-x.

Engeser, S., \& Baumann, N. (2016). Fluctuation of flow and affect in everyday life: A second look at the paradox of work. Journal of Happiness Studies, 17(1), 105-124. https://doi.org/10.1007/s10902-014-9586-4.

Hamaker, E. L. (2012). Why researchers should think "within-person": A paradigmatic rationale. In M. R. Mehl \& T. S. Conner (Eds.), Handbook of research methods for studying daily life (pp. 43-61). New York, NY: Guilford Press.

Hogarth, R. M., Portell, M., \& Cuxart, A. (2007). What risks do people perceive in everyday life? A perspective gained from the experience sampling method (ESM). Risk Analysis, 27(6), 1427-1439. https://doi.org/10. 1111/j.1539-6924.2007.00978.x.

Hu, L.-T., \& Bentler, P. M. (1999). Cutoff criteria for fit indexes in covariance structure analysis: Conventional criteria versus new alternatives. Structural Equation Modeling, 6(1), 1-55. https://doi.org/10.1080/10705 519909540118.

Hudson, W. N., Lucas, R. E., \& Donnellan, M. B. (2017). Day-to-day affect is surprisingly stable: A 2-year longitudinal study of well-being. Social Psychological and Personality Science, 8(1), 45-54. https://doi. org/10.1177/1948550616662129.

Kahneman, D., Krueger, A. B., Schkade, D. A., Schwarz, N., \& Stone, A. A. (2004). A survey method for characterizing daily life experience: The day reconstruction method. Science, 306(5702), 1776-1780. https:// doi.org/10.1126/science.1103572.

Knabe, A., Rätzel, S., Schöb, R., \& Weimann, J. (2010). Dissatisfied with life but having a good day: Timeuse and well-being of the unemployed. The Economic Journal, 120(547), 867-889. https://doi.org/10.111 1/j.1468-0297.2009.02347.x.

Kunzmann, U., Richter, D., \& Schmukle, S. C. (2013). Stability and change in affective experience across the adult life span: Analyses with a national sample from Germany. Emotion, 13(6), 1086-1095. https://doi. org/10.1037/a0033572.

Lazarus, R. S. (1991). Emotion and adaptation. New York: Oxford University Press.

LePine, J. A., Podsakoff, N. P., \& LePine, M. A. (2005). A meta-analytic test of the challenge stressor-hindrance stressor framework: An explanation for the inconsistent relationships among the stressors and performance. Academy of Management Journal, 48(5), 764-775. https://doi.org/10.5465/AMJ.2005.18803921. 
Lüdtke, O., Marsh, H. W., Robitzsch, A., Trautwein, U., Asparouhov, T., \& Muthén, B. (2008). The multilevel latent covariate model: A new, more reliable approach to group-level effects in contextual studies. Psychological Methods, 13(3), 203-229. https://doi.org/10.1037/a0012869.

Marsh, H. W., Lüdtke, O., Robitzsch, A., Trautwein, U., Asparouhov, T., Muthén, B., et al. (2009). Doublylatent models of school contextual effects: Integrating multilevel and structural equation approaches to control measurement and sampling error. Multivariate Behavioral Research, 44(6), 764-802. https://doi. org/10.1080/00273170903333665.

Molenaar, P. C., \& Campbell, C. G. (2009). The new person-specific paradigm in psychology. Current Directions in Psychological Science, 18(2), 112-117. https://doi.org/10.1111/j.1467-8721.2009.01619.x.

Muthén, L. K., \& Muthén, B. O. (1998-2015). Mplus user's guide (7th ed.). Los Angelos, CA: Muthen \& Muthen.

Nelson, S. K., Kushlev, K., English, T., Dunn, E. W., \& Lyubomirsky, S. (2013). In defense of parenthood: Children are associated with more joy than misery. Psychological Science, 24(1), 3-10. https://doi. org/10.1177/0956797612447798.

Oerlemans, W. G. M., Bakker, A. B., \& Veenhoven, R. (2011). Finding the key to happy aging: A day reconstruction study of happiness. The Journals of Gerontology: Psychological Sciences, 66B(6), 1-10. https:// doi.org/10.1093/geronb/gbr040.

Oishi, S., Kurtz, J. L., Miao, F. F., Park, J., \& Whitchurch, E. (2011). The role of familiarity in daily wellbeing: Developmental and cultural variation. Developmental Psychology, 57(6), 1750-1756. https://doi. org/10.1037/a0025305.

Richter, D., \& Schupp, J. (2015). The SOEP innovation sample (SOEP IS). Schmollers Jahrbuch, 135, 389-400.

Russell, J. A. (1980). A circumplex model of affect. Journal of Personality and Social Psychology, 39(6), 1161-1178.

Russell, J. A. (2003). Core affect and the psychological construction of emotion. Psychological Review, 110(2), 145-172. https://doi.org/10.1037/0033-295X.110.1.145.

Russell, J. A., \& Feldman Barrett, L. (1999). Core affect, prototypical emotional episodes, and other things called emotion: Dissecting the elephant. Journal of Personality and Social Psychology, 76(5), 805-819. https://doi.org/10.1037/0022-3514.76.5.805.

Satorra, A., \& Bentler, P. M. (2001). A scaled difference Chi square test statistic for moment structure analysis. Psychometrika, 66(4), 507-514. https://doi.org/10.1007/BF02296192.

Scherer, K. R. (2001). Appraisal considered as a process of multi-level sequential checking. In K. R. Scherer, A. Schorr, \& T. Johnstone (Eds.), Appraisal processes in emotion: Theory, methods, research (pp. 92-120). New York: Oxford University Press.

Schimmack, U. (1999). Strukturmodelle der Stimmungen: Rückschau, Rundschau und Ausschau [Structural models of mood: Review, overview, and outlook]. Psychologische Rundschau, 50(2), 90-97.

Shiffman, S., Stone, A. A., \& Hufford, M. R. (2008). Ecological momentary assessment. Annual Review of Clinical Psychology, 4, 1-32. https://doi.org/10.1146/annurev.clinpsy.3.022806.091415.

Stoeber, J., \& Janssen, D. P. (2011). Perfectionism and coping with daily failures: Positive reframing helps achieve satisfaction at the end of the day. Anxiety Stress and Coping, 24(5), 477-497. https://doi. org/10.1080/10615806.2011.562977.

Stone, A. A., Schwartz, J. E., Schkade, D., Schwarz, N., Krueger, A., \& Kahneman, D. (2006). A population approach to the study of emotion: Diurnal rhythms of a working day examined with the day reconstruction method. Emotion, 6(1), 139-149. https://doi.org/10.1037/1528-3542.6.1.139.

Struk, A., Scholer, A., \& Danckert, J. (2016). A self-regulatory approach to understanding boredom proneness. Cognition and Emotion, 30(8), 1388-1401. https://doi.org/10.1080/02699931.2015.1064363.

Vansteelandt, K., Van Mechelen, I., \& Nezlek, J. B. (2005). The co-occurrence of emotions in daily life: A multilevel approach. Journal of Research in Personality, 39(3), 325-335. https://doi.org/10.1016/j. jrp.2004.05.006.

Watson, D., \& Tellegen, A. (1985). Toward a consensual structure of mood. Psychological Bulletin, 98(2), 219235. https://doi.org/10.1037/0033-2909.98.2.219.

Zelenski, J. M., \& Larsen, R. J. (2000). The distribution of basic emotions in everyday life: A state and trait perspective from experience sampling data. Journal of Research in Personality, 34(2), 178-197. https ://doi.org/10.1006/jrpe.1999.2275. 